\title{
Two descent statistics over 321-avoiding centrosymmetric involutions
}

\author{
Marilena Barnabei Flavio Bonetti* \\ Dipartimento di Matematica \\ Università di Bologna \\ Italy \\ \{marilena.barnabei,flavio.bonetti\}@unibo.it \\ Sergi Elizalde ${ }^{\dagger}$ \\ Department of Mathematics \\ Dartmouth College \\ New Hampshire, USA \\ sergi.elizalde@dartmouth.edu \\ Matteo Silimbani \\ Scuola secondaria di Primo Grado M. Marinelli \\ Italy \\ matteo.silimbani4@unibo.it
}

Submitted: Sep 1, 2015; Accepted: Feb 9, 2016; Published: Feb 19, 2016

Mathematics Subject Classifications: 05A05, 05A19; 05A17; 05A15; 05A30; 05A10

\begin{abstract}
Centrosymmetric involutions in the symmetric group $\mathcal{S}_{2 n}$ are permutations $\pi$ such that $\pi=\pi^{-1}$ and $\pi(i)+\pi(2 n+1-i)=2 n+1$ for all $i$, and they are in bijection with involutions of the hyperoctahedral group. We describe the distribution of some natural descent statistics on 321-avoiding centrosymmetric involutions, including the number of descents in the first half of the involution, and the sum of the positions of these descents. Our results are based on two new bijections, one between centrosymmetric involutions in $\mathcal{S}_{2 n}$ and subsets of $\{1, \ldots, n\}$, and another one showing that certain statistics on Young diagrams that fit inside a rectangle are equidistributed. We also use the latter bijection to refine a known result stating that the distribution of the major index on 321-avoiding involutions is given by the $q$-analogue of the central binomial coefficients.
\end{abstract}

\section{Introduction}

The hyperoctahedral group $B_{n}$ is isomorphic to a subgroup of the symmetric group $\mathcal{S}_{2 n}$, namely that of centrosymmetric permutations. We say that a permutation $\pi \in \mathcal{S}_{m}$ is centrosymmetric if $\pi(i)+\pi(m+1-i)=m+1$ for every $i=1, \ldots, m$. There are several ways to define a bijection between the set $B_{n}$ and the set of centrosymmetric

\footnotetext{
*Partially supported by University of Bologna, funds for selected research topics and by P.R.I.N. of M.I.U.R., Italy

${ }^{\dagger}$ Partially supported by grant \#280575 from the Simons Foundation and by grant H98230-14-1-0125 from the NSA.
} 
permutations in $\mathcal{S}_{2 n}$. Such a bijection appears in [7], and we describe a similar one in Section 5 .

Aside from their connection with $B_{n}$, centrosymmetric permutations are interesting in their own right. For instance, it is well known that a permutation is centrosymmetric if and only if the two standard Young tableaux corresponding to $\pi$ via the RobinsonSchensted algorithm are fixed under the Schützenberger involution (see [13] and 9] for details). In a different context, the permutation matrices corresponding to centrosymmetric permutations are the extreme points of a convex subset of $n^{2}$-dimensional Euclidean space, which is characterized in [6] by a simple set of linear inequalities.

In recent years, the study of forbidden patterns has been extended to the hyperoctahedral group $B_{n}$, the natural $B$-analogue of the symmetric group. Elements of $B_{n}$ are sometimes called signed permutations. In [17], Stembridge used signed pattern avoidance to give a characterization of the so-called fully commutative top elements of the hyperoctahedral group, which are elements having an interesting algebraic property. The sets of signed permutations avoiding signed patterns of length 2 were completely characterized in [14] and [10], and the cardinalities of these sets were computed.

There is also some work in the literature on the distribution of permutation statistics both over centrosymmetric permutations and the hyperoctahedral group, as well as over subsets of these sets. For example, in [2] the authors determine the descent distribution over the set of centrosymmetric involutions, while the same distribution over centrosymmetric permutations that avoid a pattern of length 3 is given in [3]. More recently, Biagioli et al. studied the distribution of the descent number and the major index both over the hyperoctahedral group [5] and over the set of its fully commutative involutions [4].

Involutions in $\mathcal{S}_{m}$ that avoid the pattern 321 are particularly well behaved, and the distribution of descents sets over them has interesting connections with the theory of partitions, as shown in [1].

In this paper we focus on centrosymmetric 321-avoiding involutions, which we denote by $\mathcal{I}_{m}^{C}(321)$, and we study the distribution of some descent statistics over them. Identifying $B_{n}$ with centrosymmetric permutations in $\mathcal{S}_{2 n}$, the property of being an involution is preserved. The condition of avoiding 321 can also be translated in terms of avoidance of some signed patterns in $B_{n}$.

On one hand, we determine the descent polynomial on $\mathcal{I}_{2 n}^{C}(321)$, showing that

$$
\sum_{\pi \in \mathcal{I}_{2 n}^{C}(321)} q^{\operatorname{des}(\pi)}=(1+q)^{n}
$$

Recall that the major index is defined to be the sum of the descent positions of a permutation. We observe that, if $\pi \in \mathcal{I}_{m}^{C}(321)$ has a descent at position $i$, then it has also a descent at position $m-i$. Hence, the classical major index of $\pi$ is simply a multiple of its descent number. For this reason, in addition to whole number of descents we consider two more statistics, which we denote $\mathrm{des}^{+}$and $\mathrm{maj}^{+}$. They are defined as the number of descents in positions $1, \ldots,\lfloor m / 2\rfloor$ and the sum of their positions, respectively. When $m$ is odd, these statistics reduce to known statistics on 321-avoiding involutions studied in [1, so we will focus on the case that $m=2 n$.

An important ingredient in our study of these distributions is the surprising fact that every involution $\pi \in \mathcal{I}_{2 n}^{C}(321)$ is uniquely determined by its excedances in the first $n$ 
positions, and the descent set of $\pi$ can be easily read from these excedances. This will allow us to obtain the following generating polynomials:

$$
\sum_{\pi \in \mathcal{I}_{2 n}^{C}(321)} q^{\operatorname{des}^{+}(\pi)}=\frac{(1+\sqrt{q})^{n+1}+(1-\sqrt{q})^{n+1}}{2}, \quad \sum_{\pi \in \mathcal{I}_{2 n}^{C}(321)} q^{\operatorname{maj}^{+}(\pi)}=\sum_{h=0}^{n} q^{n-h}\left(\begin{array}{l}
n \\
h
\end{array}\right)_{q}
$$

where $\left(\begin{array}{l}n \\ h\end{array}\right)_{q}$ is the $q$-binomial coefficient. We give both recursive and bijective proofs of these results, exploiting the relationship between centrosymmetric involutions (more precisely, the sets of their excedances) and Young diagrams that fit inside a rectangular box.

Our results translate easily to 123 -avoiding centrosymmetric involutions, since these are in bijection with 321-avoiding ones via the complement operation.

One of the tools in our proofs is a bijection showing that certain statistics on Young diagrams that fit inside a rectangle are equidistributed. In Section 6 we use this bijection to generalize the main result from [1, which gives a bijection between 321-avoiding involutions and partitions whose Young diagram fits into a square box, with the property that the descent set of the involution is mapped to the so-called hook decomposition of the partition. We show that by modifying the bijection and replacing square boxes by rectangles, one can additionally keep track of the number of fixed points. In particular, we refine a result from [1] stating that the distribution of the major index over the set of 321-avoiding involutions is given by the $q$-analogue of the central binomial coefficients.

\section{Preliminaries}

A permutation $\pi \in \mathcal{S}_{m}$ is called centrosymmetric if

$$
\pi(i)+\pi(m+1-i)=m+1
$$

for every $1 \leqslant i \leqslant m$. Equivalently, $\pi$ is centrosymmetric if $\pi^{r}=\pi^{c}$, where $r$ and $c$ are the usual reverse and complement operations, respectively. We denote by $\mathcal{S}_{m}^{C}$ the set of centrosymmetric permutations in $\mathcal{S}_{m}$, and by $\mathcal{I}_{m}^{C}$ the set of involutions in $\mathcal{S}_{m}^{C}$.

We say that $\pi \in \mathcal{S}_{m}$ has a descent at position $i$, where $1 \leqslant i<m$, if $\pi(i)>\pi(i+1)$. The set of descent positions of $\pi$ is denoted by $\operatorname{Des}(\pi)$.

Moreover, we denote by $\operatorname{des}(\pi)$ the cardinality of $\operatorname{Des}(\pi)$. The sum of the entries in $\operatorname{Des}(\pi)$ is called the major index of $\pi$ :

$$
\operatorname{maj}(\pi)=\sum_{i \in \operatorname{Des}(\pi)} i
$$

For centrosymmetric permutations, the major index can be easily expressed in terms of the number of descents. Observe that for $\pi \in \mathcal{S}_{m}^{C}$, we have that $i \in \operatorname{Des}(\pi)$ if and only if $m-i \in \operatorname{Des}(\pi)$. This implies that $\operatorname{maj}(\pi)=m \operatorname{des}(\pi) / 2$. Hence, studying the usual major index statistic on centrosymmetric permutations is equivalent to studying the number of descents.

Because of the above symmetry, it makes sense to restrict to the set of descents in positions $1, \ldots,\left\lfloor\frac{m}{2}\right\rfloor$ which allow us to recover the whole set $\operatorname{Des}(\pi)$. Setting $n=\left\lfloor\frac{m}{2}\right\rfloor$, we are interested in the statistics

$$
\operatorname{Des}^{+}(\pi)=\operatorname{Des}(\pi) \cap[n], \quad \operatorname{des}^{+}(\pi)=\left|\operatorname{Des}^{+}(\pi)\right|,
$$


where we use the notation $[n]=\{1,2, \ldots, n\}$. We then have

$$
\operatorname{Des}(\pi)=\operatorname{Des}^{+}(\pi) \cup\left\{m-i: i \in \operatorname{Des}^{+}(\pi)\right\} .
$$

Note that this union is disjoint unless $m$ is even and $m / 2 \in \operatorname{Des}^{+}(\pi)$. It is now natural to define

$$
\operatorname{maj}^{+}(\pi)=\sum_{i \in \operatorname{Des}^{+}(\pi)} i
$$

We say that a permutation $\pi \in \mathcal{S}_{m}$ avoids the pattern $\tau \in \mathcal{S}_{k}$ if $\pi$ does not contain a subsequence $\pi\left(i_{1}\right) \pi\left(i_{2}\right) \ldots \pi\left(i_{k}\right)$ whose entries are in the same relative order as $\tau(1) \tau(2) \ldots \tau(k)$. Denote by $\mathcal{I}_{m}^{C}(321)$ the set of 321 -avoiding centrosymmetric involutions in $\mathcal{S}_{m}$. The cardinality of this set was found by Egge [7], who showed that

$$
\left|\mathcal{I}_{2 n+1}^{C}(321)\right|=\left(\begin{array}{c}
n \\
\left\lfloor\frac{n}{2}\right\rfloor
\end{array}\right)
$$

and

$$
\left|\mathcal{I}_{2 n}^{C}(321)\right|=2^{n} .
$$

The first main result of this paper gives the distribution of the statistics $\operatorname{des}^{+}$and $\mathrm{maj}^{+}$on $\mathcal{I}_{m}^{C}(321)$. When $m$ is odd, we will see that the distribution of these statistics on 321-avoiding centrosymmetric involutions can be easily obtained from the results in [1] about descents on 321-avoiding involutions. For this reason, in this paper we will focus on the case when $m$ is even.

We point out that, in the even case, the proof given in [7] of Equation (3) is not bijective. In Theorem 5 we will provide a bijective proof of this simple formula. This bijection will be a key ingredient in the proofs of the formulas giving the distribution of $\mathrm{des}^{+}$and $\mathrm{maj}^{+}$on $\mathcal{I}_{2 n}^{C}(321)$, which appear in Theorems 1 and 2 .

Another definition that will be useful is the notion of excedance of a permutation $\pi$, which is a position $i$ such that $\pi(i)>i$. We denote by $\operatorname{Exc}(\pi)$ the set of excedances of $\pi$. We use the notation $\left\{a_{1}, a_{2}, \ldots, a_{r}\right\}_{<}$to indicate that $a_{1}<a_{2}<\cdots<a_{r}$.

\section{The statistics des ${ }^{+}$and $\mathrm{maj}^{+}$on $\mathcal{I}_{m}^{C}(321)$}

In this section we give formulas for the generating polynomials for the statistics $\mathrm{des}^{+}$, $\mathrm{maj}^{+}$and des on $\mathcal{I}_{m}^{C}(321)$. The next three theorems summarize the results in the case that $m$ is even.

\section{Theorem 1.}

$$
\sum_{\pi \in \mathcal{I}_{2 n}^{C}(321)} q^{\mathrm{des}^{+}(\pi)}=\sum_{k \geqslant 0}\left(\begin{array}{c}
n+1 \\
2 k
\end{array}\right) q^{k}=\frac{(1+\sqrt{q})^{n+1}+(1-\sqrt{q})^{n+1}}{2} .
$$

\section{Theorem 2.}

$$
\sum_{\pi \in \mathcal{I}_{2 n}^{C}(321)} q^{\mathrm{maj}^{+}(\pi)}=\sum_{h=0}^{n}\left(\begin{array}{l}
n \\
h
\end{array}\right)_{q}+\left(q^{n}-1\right) \sum_{h=0}^{n-1}\left(\begin{array}{c}
n-1 \\
h
\end{array}\right)_{q}=\sum_{h=0}^{n} q^{n-h}\left(\begin{array}{l}
n \\
h
\end{array}\right)_{q}
$$

where $\left(\begin{array}{l}n \\ h\end{array}\right)_{q}$ is the q-binomial coefficient. 


\section{Theorem 3.}

$$
\sum_{\pi \in \mathcal{I}_{2 n}^{C}(321)} q^{\operatorname{des}(\pi)}=(1+q)^{n} .
$$

We will give two different proofs of Theorems 11 and 2: recursive ones in Section 3.2 and bijective ones in Section 4. An important ingredient in all of them is the bijection that we describe in Section 3.1 between $\mathcal{I}_{2 n}^{C}(321)$ and the family of all subsets of $[n]$, which we denote by $2^{[n]}$. The same bijection will be used in Section 3.3 to prove Theorem 3 .

When $m$ is odd, it is easy to check that every permutation $\pi \in \mathcal{I}_{2 n+1}^{C}(321)$ can be decomposed uniquely as $\pi=\alpha n+1 \alpha^{\prime}$, where $\alpha$ is an arbitrary element of $\mathcal{I}_{n}(321)$ and $\alpha^{\prime}$ is the sequence $2 n+2-\alpha(n), 2 n+2-\alpha(n-1), \ldots, 2 n+2-\alpha(1)$. Thus, $\mathcal{I}_{2 n+1}^{C}(321)$ is in bijection with $\mathcal{I}_{n}(321)$, and Equation (2) follows from [15]. Additionally, since

$$
\operatorname{des}^{+}(\pi)=\operatorname{des}(\alpha), \operatorname{maj}^{+}(\pi)=\operatorname{maj}(\alpha), \quad \text { and } \operatorname{des}(\pi)=2 \operatorname{des}(\alpha),
$$

the formulas for the generating polynomials for $\mathrm{des}^{+}, \mathrm{maj}^{+}$and des on $\mathcal{I}_{2 n+1}^{C}(321)$ follow from the results in [1] about descents on 321-avoiding involutions:

\section{Proposition 4.}

$$
\begin{aligned}
\sum_{\pi \in \mathcal{I}_{2 n+1}^{C}(321)} q^{\operatorname{des}^{+}(\pi)} & =\sum_{k=0}^{\left\lfloor\frac{n}{2}\right\rfloor}\left(\begin{array}{c}
\left\lceil\frac{n}{2}\right\rceil \\
k
\end{array}\right)\left(\begin{array}{c}
\left\lfloor\frac{n}{2}\right\rfloor \\
k
\end{array}\right) q^{k}, \\
\sum_{\pi \in \mathcal{I}_{2 n+1}^{C}(321)} q^{\operatorname{maj}^{+}(\pi)} & =\left(\begin{array}{c}
n \\
\left\lfloor\frac{n}{2}\right\rfloor
\end{array}\right)_{q}, \\
\sum_{\pi \in \mathcal{I}_{2 n+1}^{C}(321)} q^{\operatorname{des}(\pi)} & =\sum_{k=0}^{\left\lfloor\frac{n}{2}\right\rfloor}\left(\begin{array}{c}
\left\lceil\frac{n}{2}\right\rceil \\
k
\end{array}\right)\left(\begin{array}{c}
\left\lfloor\frac{n}{2}\right\rfloor \\
k
\end{array}\right) q^{2 k} .
\end{aligned}
$$

\section{$3.1 \quad$ A bijection $\mathcal{I}_{2 n}^{C}(321) \rightarrow 2^{[n]}$}

Elements of $\mathcal{I}_{2 n}^{C}$ can be interpreted as symmetric matchings on $2 n$ points. We draw matchings by placing $2 n$ points on a horizontal line, labeled from 1 to $2 n$ from left to right, where some pairs of points are matched with an arc. Given $\pi \in \mathcal{I}_{2 n}^{C}$, the corresponding matching has an arc between $i$ and $j$ whenever $\pi(i)=j$ (equivalently, $\pi(j)=i$, since $\pi$ is an involution) and $i \neq j$. The centrosymmetric condition is equivalent to the matching being symmetric, that is, invariant under relabeling the points from right to left instead.

Under this interpretation, an involution avoids 321 if and only if the corresponding matching is non-nesting, meaning that it does not contain two $\operatorname{arcs}(i, l)$ and $(j, k)$ with $i<j<k<l$, or an arc $(i, k)$ and a singleton (i.e., a fixed point of the permutation) $j$ with $i<j<k$. Thus, elements of $\mathcal{I}_{2 n}^{C}(321)$ are in bijection with symmetric non-nesting matchings of $2 n$ points.

For $\pi \in \mathcal{I}_{2 n}^{C}(321)$, define

$$
E_{\pi}=\operatorname{Exc}(\pi) \cap[n],
$$

that is, the set of excedances in the first $n$ positions. If $M$ is the matching corresponding to $\pi$, let $E_{M}$ denote the set of values in $[n]$ that are matched with a larger value. Clearly, $E_{\pi}=E_{M}$. 
Theorem 5. The map

$$
\begin{array}{cl}
\mathcal{I}_{2 n}^{C}(321) & \rightarrow 2^{[n]} \\
\pi & \mapsto E_{n}
\end{array}
$$

is a bijection. In particular, elements of $\mathcal{I}_{2 n}^{C}(321)$ are uniquely determined by their excedances in $[n]$.

Proof. By the above interpretation of $\mathcal{I}_{2 n}^{C}(321)$ as symmetric non-nesting matchings of $2 n$ points, it will be enough to show that the map $M \mapsto E_{M}$ is a bijection between such matchings and $2^{[n]}$. We do so by describing its inverse.

Given $E \subseteq[n]$, construct a matching $M_{E}$ as follows. Read the elements of $E$ in increasing order, and for each $i \in E$ that has not been matched, draw an arc from $i$ to the smallest $j \notin E$ such that $j>i$ and $j$ has not been matched yet, and symmetrically draw an arc $(2 n+1-j, 2 n+1-i)$, unless this is the same arc $(i, j)$ that was just added.

For example for $n=11$ and $E=\{1,4,5,7,8,10\} \subseteq[11]$, we get the matching

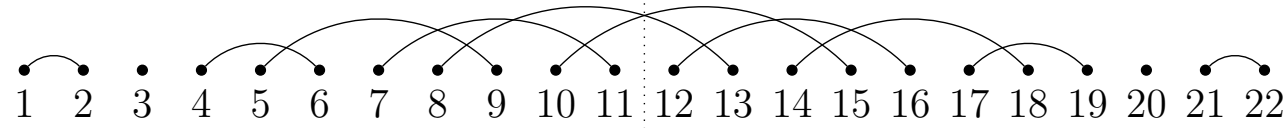

Next we show that, for any $E \subseteq[n], M_{E}$ is a well-defined symmetric non-nesting matching.

The symmetry is clear because, at any stage of the process, when adding the arcs $(i, j)$ and $(2 n+1-j, 2 n+1-i)$ (if different), the matching drawn so far is symmetric.

To see that it is well defined, we have to check that for every $i \in E$ that has not been matched, there is always an available vertex $j$ that it can be matched with. This is because since $i$ has not been matched, by symmetry neither has $2 n+1-i$, and this value is not in $E$ because it is greater than $n$, so there is always at least one available vertex. This also proves that the arcs $(i, j)$ and $(2 n+1-j, 2 n+1-i)$ added at each step satisfy $i+j \leqslant 2 n+1$, and thus $(2 n+1-j)+(2 n+1-i) \geqslant 2 n+1$.

To show that $M_{E}$ is non-nesting, suppose first for contradiction that there is a triple $i<j<k$ where $(i, k)$ is an arc and $j$ is a singleton. By symmetry, we can assume without loss of generality that $i+k \leqslant 2 n+1$, so the arc $(i, k)$ was added as the first of a pair of symmetric arcs. But then, our construction would have matched $i$ with $j$, since $j \notin E$ (because it is a singleton) and $i<j<k$.

Similarly, suppose for contradiction that there are two nested $\operatorname{arcs}(i, l)$ and $(j, k)$ with $i<j<k<l$. Again, by symmetry, we can assume without loss of generality that $i+l \leqslant 2 n+1$, by flipping both arcs if necessary. In fact, we can also assume that $j+k \leqslant 2 n+1$, because otherwise, flipping the arc $(j, k)$ we would still get two nested arcs. But then, our construction would have matched $i$ with $k$, since $k \notin E$ (because it is matched with a smaller element) and the arc $(j, k)$ had not been added at that point of the process.

Finally, we show that the map $M \mapsto E_{M}$ is a bijection between the set of symmetric non-nesting matchings of $2 n$ points and $2^{[n]}$, whose inverse is the map $E \mapsto M_{E}$. To see that it is surjective, we show that for every $E \subseteq[n]$ we have $E_{M_{E}}=E$. In other words, we show that in $M_{E}$, the points in $[n]$ matched with larger points are precisely those in $E$. Clearly, the points in $E$ are always matched with larger points, since in our construction neither of the points $j$ and $2 n+1-i$ in the pair of $\operatorname{arcs}(i, j)$ and $(2 n+1-j, 2 n+1-i)$ is 
in $E$. So it remains to show that if $2 n+1-j \leqslant n$, then $2 n+1-j \in E$. This is because if $2 n+1-j \notin E$, then, since $i<2 n+1-j<j$, the point $i$ would have been matched with $2 n+1-j$ rather than with $j$.

To see that the map $M \mapsto E_{M}$ is injective, we will show that for every $E \subseteq[n]$, the only symmetric non-nesting matching $M$ satisfying $E_{M}=E$ is the matching $M=M_{E}$. We will argue that at each step of the above construction that scans $E$ in increasing order, the only way to match $i \in E$ and preserve the non-nesting property is by matching it with the smallest $j \notin E$ such that $j>i$ and $j$ has not yet been matched. Suppose that this is not true, and consider the first time that we have some other choice for matching $i \in E$. It is clear that $i$ has to be matched with a vertex $j^{\prime} \notin E$ such that $j^{\prime}>i$ and $j^{\prime}$ has not yet been matched, so suppose we match $i$ with a vertex $j^{\prime}$ satisfying this property but not being the smallest. Then, we claim that the arc $\left(i, j^{\prime}\right)$ would be creating a nesting, which is a contradiction. Indeed, if $j$ does not become matched later on, then the singleton $j$ with the arc $\left(i, j^{\prime}\right)$ would form a nesting. If $j$ becomes matched with a point $r$, we know that $r>i$, since all the points in $E$ to the left of $i$ have been matched before $i$. If $i<r<j^{\prime}$, then the $\operatorname{arcs}\left(i, j^{\prime}\right)$ and $(r, j)$ (or $\left.(j, r)\right)$ would form a nesting. If $r>j^{\prime}$, then the conditions $j \notin E$ and $E_{M}=E$ imply that $j>n$, and so the $\operatorname{arcs}\left(i, j^{\prime}\right)$ and $(2 n+1-r, 2 n+1-j)$ (which is forced by symmetry) would form an nesting, since $i<2 n+1-r<2 n+1-j<n<j^{\prime}$, with the first inequality implied again by the fact that the points in $E$ to the left of $i$ have been matched before $i$. Clearly, once we add the $\operatorname{arc}(i, j)$, the arc $(2 n+1-j, 2 n+1-i)$ is forced by symmetry, so all the arcs in the construction of $M_{E}$ are forced.

\subsection{Recursive proofs of Theorems 1 and 2}

The set $\operatorname{Des}^{+}(\pi)$ can be easily recovered from $E_{\pi}$ as follows.

Lemma 6. For $\pi \in \mathcal{I}_{2 n}^{C}(321)$,

$$
\operatorname{Des}^{+}(\pi)=\left\{i \in[n] \mid i \in E_{\pi} \wedge i+1 \notin E_{\pi}\right\} .
$$

Proof. Let $i \in[n-1]$, and consider four possible cases:

- If $i \in E_{\pi}$ and $i+1 \notin E_{\pi}$, then $\pi(i)>i$ and $\pi(i+1) \leqslant i+1$, so $i \in \operatorname{Des}^{+}(\pi)$.

- If $i \notin E_{\pi}$ and $i+1 \in E_{\pi}$, then $\pi(i) \leqslant i$ and $\pi(i+1)>i+1$, so $i \notin \operatorname{Des}^{+}(\pi)$.

- If $\{i, i+1\} \subseteq E_{\pi}$, then $\pi(i)>i$ and $\pi(i+1)>i+1$. If $\pi(i)>\pi(i+1)$, then $\pi$ would have the 321 pattern $\pi(i) \pi(i+1) i+1$. Hence, $i \notin \operatorname{Des}^{+}(\pi)$.

- If neither $i$ nor $i+1$ are excedance positions, then $\pi(i) \leqslant i$ and $\pi(i+1) \leqslant i+1$. If $\pi(i)>\pi(i+1)$, then $\pi$ would have the 321 pattern $i+1 \pi(i) \pi(i+1)$. Hence, $i \notin \operatorname{Des}^{+}(\pi)$.

Consider now the case $i=n$, and note that $n+1 \notin E_{\pi}$. Since $\pi$ is centrosymmetric, $\pi(n)+\pi(n+1)=2 n+1$. Thus, the condition for $n \in \operatorname{Des}^{+}(\pi)$, that is, $\pi(n)>\pi(n+1)$, is equivalent to $\pi(n)>n$, which is the condition for $n \in E_{\pi}$. 
Lemma 6 suggests the following extension of the notion of descent set, descent number, and major index to subsets of $[n]$ :

$$
\begin{aligned}
\operatorname{Des}(E) & =\{i \mid i \in E \wedge i+1 \notin E\}, \\
\operatorname{des}(E) & =|\operatorname{Des}(E)|, \\
\operatorname{maj}(E) & =\sum_{i \in \operatorname{Des}(E)} i .
\end{aligned}
$$

With this notation, $\operatorname{Des}^{+}(\pi)=\operatorname{Des}\left(E_{\pi}\right)$, and so $\operatorname{des}^{+}(\pi)=\operatorname{des}\left(E_{\pi}\right)$ and $\operatorname{maj}^{+}(\pi)=$ $\operatorname{maj}\left(E_{\pi}\right)$ for all $\pi \in \mathcal{I}_{2 n}^{C}(321)$.

Define the the polynomials

$$
d_{n}(q)=\sum_{\pi \in \mathcal{I}_{2 n}^{C}(321)} q^{\mathrm{des}^{+}(\pi)}, \quad p_{n}(q)=\sum_{\pi \in \mathcal{I}_{2 n}^{C}(321)} q^{\mathrm{maj}^{+}(\pi)} .
$$

To prove Theorems 1 and 2 , we will show that $d_{n}(q)$ and $p_{n}(q)$ satisfy the same recurrences as the respective expressions given in these two theorems.

Lemma 7. For $n \geqslant 2$, the polynomials $d_{n}(q)$ and $p_{n}(q)$ satisfy the following recurrences:

$$
\begin{gathered}
d_{n}(q)=2 d_{n-1}(q)+(q-1) d_{n-2}(q), \\
p_{n}(q)=(1+q) p_{n-1}(q)+\left(q^{n}-q\right) p_{n-2}(q) .
\end{gathered}
$$

Proof. Theorem 5 and Lemma 6 allow us to rewrite the two polynomials $d_{n}(q)$ and $p_{n}(q)$ as follows:

$$
d_{n}(q)=\sum_{E \subseteq[n]} q^{\operatorname{des}(E)}, \quad p_{n}(q)=\sum_{E \subseteq[n]} q^{\operatorname{maj}(E)} .
$$

Consider now a subset $E \subseteq[n]$. There are three possible cases:

- If $n \notin E$, then $E$ is a subset of $[n-1]$ with the same descent set and major index.

- If $n \in E$ and $n-1 \notin E$, then removing $n$ from $E$ yields a subset $E^{\prime}$ of $[n-2]$ with $\operatorname{Des}\left(E^{\prime}\right)=\operatorname{Des}(E) \backslash\{n\}$ and $\operatorname{maj}\left(E^{\prime}\right)=\operatorname{maj}(E)-n$.

- If $n, n-1 \in E$, then removing $n$ from $E$ yields a subset $E^{\prime} \subseteq[n-1]$ with $\operatorname{Des}\left(E^{\prime}\right)=$ $\operatorname{Des}(E) \backslash\{n\} \cup\{n-1\}$ and $\operatorname{maj}\left(E^{\prime}\right)=\operatorname{maj}(E)-1$. Since $E^{\prime}$ contains $n-1$, the subsets $E^{\prime}$ obtained in this way are those subsets of $[n-1]$ which are not a subsets of $[n-2]$.

These considerations give

$$
\begin{gathered}
d_{n}(q)=d_{n-1}(q)+q d_{n-2}(q)+\left(d_{n-1}(q)-d_{n-2}(q)\right), \\
p_{n}(q)=p_{n-1}(q)+q^{n} p_{n-2}(q)+q\left(p_{n-1}(q)-p_{n-2}(q)\right),
\end{gathered}
$$

which are equivalent to the above recurrences. 
Theorem 1 follows now from Lemma 7. Indeed, a routine computation shows that the polynomial

$$
\sum_{k \geqslant 0}\left(\begin{array}{c}
n+1 \\
2 k
\end{array}\right) q^{k}
$$

satisfies the same recurrence as $d_{n}(q)$ with the same initial conditions.

In order to prove Theorem 2 , we need a few more definitions. Let $R_{a, b}$ denote an $a \times b$ rectangle, and let $\mathcal{Y}_{a, b}$ denote the set of Young diagrams that fit inside of $R_{a, b}$ (where the convention is that diagrams are placed touching the upper and left sides of the rectangle). By considering the southeast boundary of the Young diagram, we identify $\mathcal{Y}_{a, b}$ with the set of paths with steps $N$ and $E$ from $(0,0)$ to $(b, a)$. It is well known that $\left|\mathcal{Y}_{a, b}\right|=\left(\begin{array}{c}a+b \\ a\end{array}\right)$.

For every positive integer $n$, we can represent the subsets of $[n]$ as lattice paths with unit steps $N$ and $E$ starting at the origin, where the $i$ th step is $N$ if and only if $i$ belongs to the subset. Let $\mathcal{A}_{n}$ denote the set of all lattice paths with $n$ steps $N$ and $E$ starting at the origin. Note that $\left|\mathcal{A}_{n}\right|=2^{n}$ and

$$
\mathcal{A}_{n}=\bigcup_{a+b=n} \mathcal{Y}_{a, b}
$$

Consider now the set $\mathcal{Y}_{a, b}^{E}$ of paths with steps $N$ and $E$ from $(0,0)$ to $(b+1, a)$ starting with an $E$ step. The set $\mathcal{Y}_{a, b}^{E}$ is obviously in bijection with $\mathcal{Y}_{a, b}$. Define

$$
\mathcal{A}_{n}^{E}=\bigcup_{a+b=n} \mathcal{Y}_{a, b}^{E}
$$

and

$$
r_{n}(q)=\sum_{P \in \mathcal{A}_{n}^{E}} q^{\operatorname{area}(P)},
$$

where $\operatorname{area}(P)$ is the area of the Young diagram corresponding to $P$. Then,

$$
r_{n}(q)=\sum_{a=0}^{n} \sum_{P \in \mathcal{Y}_{a, n-a}^{E}} q^{\operatorname{area}(P)}=\sum_{a=0}^{n} q^{n-a} \sum_{\bar{P} \in \mathcal{Y}_{a, n-a}} q^{\operatorname{area}(\bar{P})}
$$

where $\bar{P}$ is the path obtained from $P$ by deleting the first step. This last espression equals

$$
\sum_{a=0}^{n} q^{n-a}\left(\begin{array}{l}
n \\
a
\end{array}\right)_{q}
$$

using the well-known fact [16] that the coefficient of $q^{r}$ in the $q$-binomial coefficient $\left(\begin{array}{l}n \\ a\end{array}\right)_{q}$ equals the number of partitions of $r$ whose Young diagram is in $\mathcal{Y}_{a, n-a}$.

To prove Theorem 2, it is now enough to show is that the polynomials $r_{n}(q)$ satisfy the same recurrence as the one given in Lemma 7 for $p_{n}(q)$. It is clear that their initial values coincide.

Proposition 8. For $n \geqslant 2$, the polynomials $r_{n}(q)$ defined in Equation (5) satisfy the recurrence

$$
r_{n}(q)=(1+q) r_{n-1}(q)+\left(q^{n}-q\right) r_{n-2}(q)
$$


Proof. Consider a path $P \in \mathcal{A}_{n}^{E}$. Once again, there are three possible cases, as illustrated in Figure 1:

- If $P$ ends with an $E$ step, remove it, getting an element of $\mathcal{A}_{n-1}^{E}$ with the same area.

- If $P$ ends with an $N$ step and begins with $E E$, remove the first and last step from $P$, getting an element of $\mathcal{A}_{n-2}^{E}$ whose area has decreased by $n$.

- If $P$ ends with an $N$ step and begins with $E N$, remove the second step in $P$, getting an element of $\mathcal{A}_{n-1}^{E}$ whose area has decreased by 1 . The resulting path is not an arbitrary element of $\mathcal{A}_{n-1}^{E}$, but one ending with an $N$ step. Such paths contribute $r_{n-1}(q)-r_{n-2}(q)$ to the generating polynomial, since paths in $\mathcal{A}_{n-1}^{E}$ ending with an $E$ step are equivalent to paths in $\mathcal{A}_{n-2}^{E}$.

These considerations give

$$
r_{n}(q)=r_{n-1}(q)+q^{n} r_{n-2}(q)+q\left(r_{n-1}(q)-r_{n-2}(q)\right),
$$

which is equivalent to the stated recurrence.
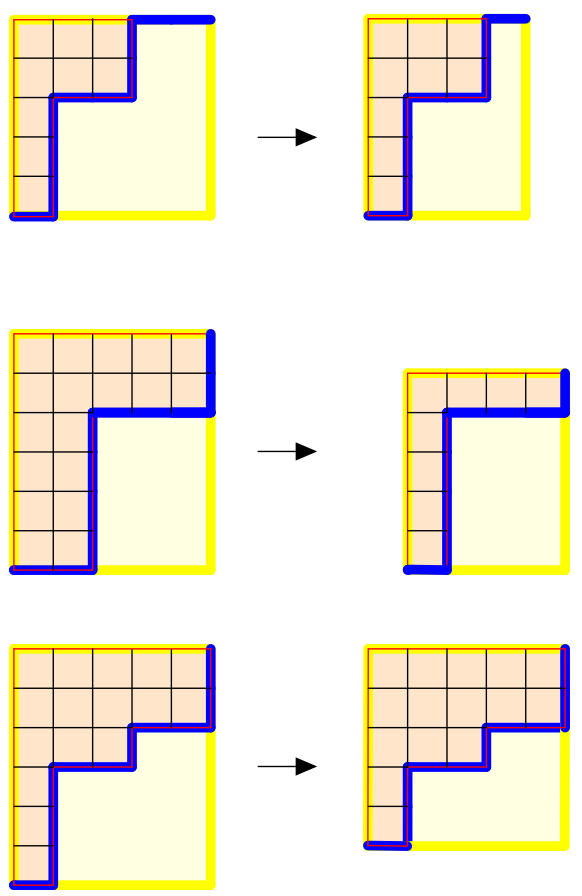

Figure 1: The three cases in the proof of Proposition 8 .

\subsection{Proof of Theorem 3}

To obtain the generating polynomial for the statistic des on $\mathcal{I}_{2 n}^{C}(321)$, we will combine Lemma 6 with the following simple fact. 
Lemma 9. Let $\pi \in \mathcal{I}_{2 n}^{C}(321)$ and recall the definition of $E_{\pi}$ from Equation (4). Then,

$$
\operatorname{des}(\pi)= \begin{cases}2 \operatorname{des}^{+}(\pi)-1=2 \operatorname{des}\left(E_{\pi}\right)-1 & \text { if } n \in E_{\pi}, \\ 2 \operatorname{des}^{+}(\pi)=2 \operatorname{des}\left(E_{\pi}\right) & \text { otherwise. }\end{cases}
$$

Proof. By Equation (1), Des $(\pi)=\operatorname{Des}^{+}(\pi) \cup\left\{2 n-i: i \in \operatorname{Des}^{+}(\pi)\right\}$ and this union is disjoint unless $n \in \operatorname{Des}^{+}(\pi)$, or equivalently $n \in E_{\pi}$. The equality $\operatorname{des}^{+}(\pi)=\operatorname{des}\left(E_{\pi}\right)$ follows from Lemma 6 .

Proof of Theorem 3. By Theorem 5 involutions in $\mathcal{I}_{2 n}^{C}(321)$ are in bijection with subsets of $[n]$. Let $\pi \in \mathcal{I}_{2 n-2}^{C}(321)$ and let $E=E_{\pi} \subseteq[n-1]$ be the corresponding subset. We build two involutions in $\mathcal{I}_{2 n}^{C}(321)$ as follows: let $\tilde{\pi}$ be the involution corresponding to $E$, seen as a subset of $[n]$; and let $\hat{\pi}$ be the involution corresponding to the set $E \cup\{n\}$. Setting $t=\operatorname{des}(E)$ and applying Lemma 9, we have

- If $n-1 \in E$, then

$$
\operatorname{des}(\pi)=2 t-1, \quad \operatorname{des}(\tilde{\pi})=2 t, \quad \operatorname{des}(\hat{\pi})=2 t-1 .
$$

- If $n-1 \notin E$, then

$$
\operatorname{des}(\pi)=2 t, \quad \operatorname{des}(\tilde{\pi})=2 t, \quad \operatorname{des}(\hat{\pi})=2 t+1 .
$$

This implies that

$$
\sum_{\pi \in \mathcal{I}_{2 n}^{C}(321)} q^{\operatorname{des}(\pi)}=(1+q) \sum_{\pi \in \mathcal{I}_{2 n-2}^{C}(321)} q^{\operatorname{des}(\pi)} .
$$

Since $\sum_{\pi \in \mathcal{I}_{2}^{C}(321)} q^{\operatorname{des}(\pi)}=1+q$, we get formula by induction.

\section{Bijective proofs of Theorems 1 and 2}

In this section we show that Theorems 1 and 2 can be also proved bijectively. These bijective proofs are based upon a more careful analysis of the connections between lattice paths and integer partitions.

Define a peak of a path in $\mathcal{A}_{n}$ to be an occurrence of $N E$, or equivalently the vertex in the middle of such an occurrence. If we label the vertices of a path $P \in \mathcal{A}_{n}$ from 0 to $n$ starting at the origin, the peak set of $P$, denoted $\operatorname{Peak}(P)$, is the set of labels of the vertices that are peaks in $P$. Let $\operatorname{Peak}^{*}(P)=\operatorname{Peak}(P E)$, where $P E$ is the path obtained from $P$ by appending a step $E$ at the end. Note that

$$
\operatorname{Peak}^{*}(P)= \begin{cases}\operatorname{Peak}(P) \cup\{n\} & \text { if } P \text { ends with an } N \text { step, } \\ \operatorname{Peak}(P) & \text { otherwise. }\end{cases}
$$

Given a $P \in \mathcal{A}_{n}$, and thinking of it as a the south east edge of a Young diagram $\lambda$, we define its hook decomposition $\operatorname{HD}(P)=\left\{i_{1}, i_{2}, \ldots, i_{k}\right\}$ as follows. The number of entries $k$ is the length of the side of the Durfee square of $\lambda$, that is, the largest value such 
that $\lambda_{k} \geqslant k$. The largest entry $i_{k}$ is the number of boxes in the largest hook of $\lambda$, which consists of the first column and first row of its Young diagram. Now remove the largest hook of $\lambda$ and define $i_{k-1}$ to be the number of boxes in the largest hook of the remaining Young diagram. Similarly, the remaining entries $i_{j}$ are defined recursively by peeling off hooks in the Young diagram.

Define

$$
\operatorname{HD}^{*}(P)= \begin{cases}\operatorname{HD}(P) \cup\{n\} & \text { if } P \text { begins with an } N \text { step, } \\ \operatorname{HD}(P) & \text { otherwise. }\end{cases}
$$

Lemma 10. There is a bijection $g: \mathcal{Y}_{a, b} \rightarrow \mathcal{Y}_{a, b}$ such that

$$
\operatorname{Peak}(P)=\mathrm{HD}(g(P)) \quad \text { and } \operatorname{Peak}^{*}(P)=\operatorname{HD}^{*}(g(P)) .
$$

Proof. The map $g$ that we describe here is a generalization of the bijection $\psi^{-1}$ from [1, Lemma 3.5] to arbitrary rectangles. Given $P \in \mathcal{Y}_{a, b}$, let $\left(x_{j}, y_{j}\right), 1 \leqslant j \leqslant k$ the coordinates of its $k$ peaks, where $0 \leqslant x_{1}<\cdots<x_{k} \leqslant b-1$ and $1 \leqslant y_{1}<\cdots<y_{k} \leqslant a$. Then

$$
g(P)=R_{a} R_{a-1} \ldots R_{1} S_{1} S_{2} \ldots S_{b},
$$

where $R_{y_{j}}=E$ for $1 \leqslant j \leqslant k$ and $R_{i}=N$ otherwise, and $S_{x_{j}+1}=N$ for $1 \leqslant j \leqslant k$ and $S_{i}=E$ otherwise.

Clearly, $g(P) \in \mathcal{Y}_{a, b}$ because it has $a-k+k N$ steps and $b-k+k E$ steps. It is easy to check from the construction that

$$
\operatorname{HD}(g(P))=\left\{x_{1}+y_{1}, x_{2}+y_{2}, \ldots, x_{k}+y_{k}\right\}=\operatorname{Peak}(P) .
$$

Also, the last step of $P$ is an $N$ if and only if the first step of $g(P)$ is an $N$, and so $\operatorname{HD}^{*}(g(P))=\operatorname{Peak}^{*}(P)$ as well.

To see that the map $g$ is a bijection, note that for any path $Q=R_{a} R_{a-1} \ldots R_{1} S_{1} S_{2} \ldots S_{b} \in$ $\mathcal{Y}_{a, b}$, the positions of the $N$ and $E$ steps can be used to determine the coordinates $\left(x_{j}, y_{j}\right)$ of the peaks of the path $g^{-1}(Q)$.

With some abuse of notation, we also denote by $g$ the bijection from $\mathcal{A}_{n}=\bigcup_{a+b=n} \mathcal{Y}_{a, b}$ to itself.

By Theorem 5, $\mathcal{I}_{2 n}^{C}(321)$ is in bijection with the family of all subsets of $[n]$. Let $f$ be the bijection between $\mathcal{I}_{2 n}^{C}(321)$ and $\mathcal{A}_{n}$ given at the beginning of Section 3 together with the interpretation of subsets of $[n]$ as lattice paths described above. For $\pi \in \mathcal{I}_{2 n}^{C}(321)$, we have

$$
\operatorname{Des}^{+}(\pi)=\operatorname{Peak}^{*}(f(\pi))
$$

In particular, $\operatorname{des}^{+}(\pi)=\left|\operatorname{Peak}^{*}(f(\pi))\right|$, and $\mathrm{maj}^{+}(\pi)=\sum_{i \in \operatorname{Peak}^{*}(f(\pi))} i$.

Bijective proof of Theorem 1. By Equation (6),

$$
\sum_{\pi \in \mathcal{I}_{2 n}^{C}(321)} q^{\text {des }^{+}(\pi)}=\sum_{P \in \mathcal{A}_{n}} q^{\mid \text {Peak }^{*}(P) \mid} .
$$

To find the coefficient of $q^{k}$, it is enough to count the number of paths $P \in \mathcal{A}_{n}$ with $\left|\operatorname{Peak}^{*}(P)\right|=k$. These are precisely paths of the form

$$
P=E^{i_{1}} N^{j_{1}} N E E^{i_{2}} N^{j_{2}} N E \ldots N E E^{i_{k}} N^{j_{k}} N E^{i_{k+1}},
$$

where $i_{\ell}, j_{\ell} \geqslant 0$ for all $\ell$, and $\sum_{\ell} i_{\ell}+\sum_{\ell} j_{\ell}=n-2 k+1$. Thus, the number of such paths is the number of ways to put $n-2 k+1$ balls into $2 k+1$ bins, which is $\left(\begin{array}{c}n+1 \\ 2 k\end{array}\right)$. 
Bijective proof of Theorem 2. Consider the composition

$$
\mathcal{I}_{2 n}^{C}(321) \stackrel{f}{\longrightarrow} \mathcal{A}_{n} \stackrel{g}{\longrightarrow} \mathcal{A}_{n} .
$$

For $\pi \in \mathcal{I}_{2 n}^{C}(321)$, Equation (6) and Lemma 10 imply that

$$
\operatorname{Des}^{+}(\pi)=\operatorname{Peak}^{*}(f(\pi))=\operatorname{HD}^{*}(g(f(\pi)) .
$$

Note that for $P \in \mathcal{A}_{n}$, we have $\sum_{i \in \mathrm{HD}(P)} i=\operatorname{area}(P)$, the area of the Young diagram of which $P$ is the south east border. Thus, by the definition of HD*,

$$
\sum_{i \in \mathrm{HD}^{*}(P)} i= \begin{cases}\operatorname{area}(P)+n & \text { if } P \text { begins with an } N \text { step } \\ \operatorname{area}(P) & \text { otherwise. }\end{cases}
$$

It follows that

$$
\sum_{\pi \in \mathcal{I}_{2 n}^{C}(321)} q^{\mathrm{maj}^{+}(\pi)}=\sum_{P \in \mathcal{A}_{n}} q^{\sum_{i \in \mathrm{HD}^{*}(P)}{ }^{i}}=\sum_{P \in \mathcal{A}_{n}} q^{\operatorname{area}(P)}+\left(q^{n}-1\right) \sum_{Q \in \mathcal{A}_{n-1}} q^{\operatorname{area}(Q)},
$$

by separating paths that begin with an $N$ step and writing them as $P=N Q$, with $Q \in \mathcal{A}_{n-1}$ and area $(P)=\operatorname{area}(Q)$. By definition of the $q$-binomial coefficients, we get

$$
\sum_{\pi \in \mathcal{I}_{2 n}^{C}(321)} q^{\mathrm{maj}^{+}(\pi)}=\sum_{h=0}^{n}\left(\begin{array}{l}
n \\
h
\end{array}\right)_{q}+\left(q^{n}-1\right) \sum_{h=0}^{n-1}\left(\begin{array}{c}
n-1 \\
h
\end{array}\right)_{q} .
$$

To obtain the equivalent expression $\sum_{h=0}^{n} q^{n-h}\left(\begin{array}{l}n \\ h\end{array}\right)_{q}$, we need one more bijection. For any $P \in \mathcal{Y}_{n-h, h}$, let $P^{\prime} \in \mathcal{Y}_{n-h, h}$ be the path obtained from $P$ by moving the first step of $P$ to the end. Then

$$
\operatorname{area}\left(P^{\prime}\right)= \begin{cases}\operatorname{area}(P)+h & \text { if } P \text { begins with an } N \text { step, } \\ \operatorname{area}(P)-(n-h) & \text { otherwise. }\end{cases}
$$

Combining this with equation (7), we see that $\sum_{i \in \mathrm{HD}^{*}(P)} i=\operatorname{area}\left(P^{\prime}\right)+n-h$. Thus,

$$
\sum_{\pi \in \mathcal{I}_{2 n}^{C}(321)} q^{\mathrm{maj}^{+}(\pi)}=\sum_{h=0}^{n} \sum_{P \in \mathcal{Y}_{n-h, h}} q^{\sum_{i \in \mathrm{HD}^{*}(P)} i}=\sum_{h=0}^{n} \sum_{P^{\prime} \in \mathcal{Y}_{n-h, h}} q^{\operatorname{area}\left(P^{\prime}\right)+n-h}=\sum_{h=0}^{n} q^{n-h}\left(\begin{array}{l}
n \\
h
\end{array}\right)_{q}
$$

\section{Connections with the hyperoctahedral group}

Recall that the hyperoctahedral group $B_{n}$ is the set of bijections $\pi$ from the set $\{-n, \ldots,-2,-1,1,2, \ldots, n\}$ to itself such that $\pi(-i)=-\pi(i)$ for $1 \leqslant i \leqslant n$. In particular, $\pi$ is described by the sequence $\pi=\pi(1) \ldots \pi(n)$, sometimes called a signed permutation. We denote its absolute value by $|\pi|=|\pi(1)| \ldots|\pi(n)| \in \mathcal{S}_{n}$. It will be convenient to denote negative entries $-a$ by $\bar{a}$. 
The group of centrosymmetric permutations $\mathcal{S}_{2 n}^{C}$ corresponds bijectively to the hyperoctahedral group $B_{n}$ via the map $\Theta: \mathcal{S}_{2 n}^{C} \rightarrow B_{n}$ that associates a permutation $\pi \in \mathcal{S}_{2 n}^{C}$ to the signed permutation $\tilde{\pi}=\Theta(\pi) \in B_{n}$ defined by

$$
\tilde{\pi}(i)= \begin{cases}\pi(n+i)-n & \text { if } \pi(n+i)>n \\ \pi(n+i)-n-1 & \text { otherwise, }\end{cases}
$$

for $1 \leqslant i \leqslant n$ (a similar bijection appears in [7]).

For example, if $\pi=24863157 \in \mathcal{S}_{2 n}^{C}$, then $\Theta(\pi)=\overline{2} \overline{4} 13 \in B_{n}$. Drawing a permutation $\pi \in \mathcal{S}_{n}$ as an $n \times n$ array with a marker in column $i$ and row $\pi(i)$ for each $i$, where rows and columns are labeled by $1,2, \ldots, n$ starting from the bottom left, the operation $\Theta$ amounts to relabeling the rows and columns by $-n, \ldots,-1,1, \ldots, n$, as shown in Figure 2. From this description, it is immediate that $\pi$ is an involution if and only if so is $\tilde{\pi}$ (meaning that $\tilde{\pi}(\tilde{\pi}(i))=i$ for every $i$ ), since involutions correspond to arrays that are symmetric with respect to the diagonal.
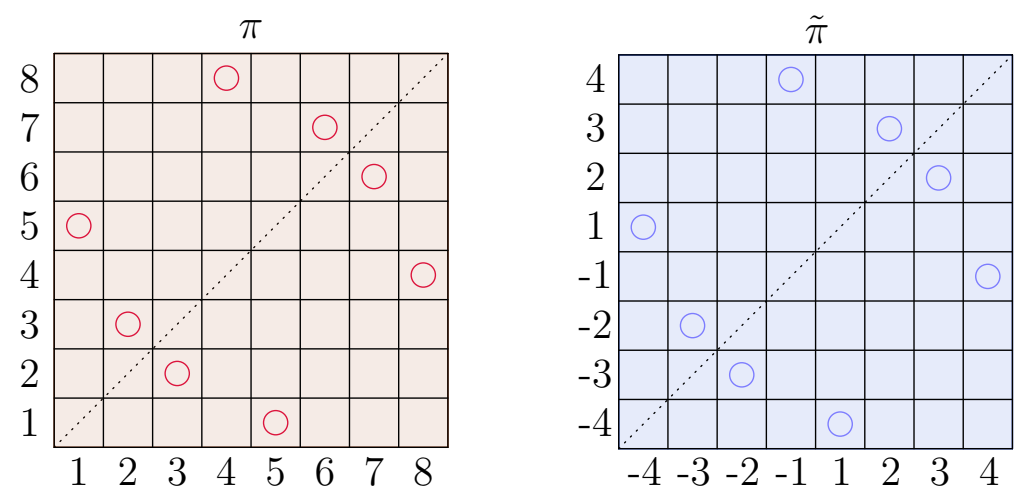

Figure 2: An involution $\pi=53281764 \in \mathcal{S}_{8}^{C}$ and the corresponding signed involution $\tilde{\pi}=\overline{4} 32 \overline{1} \in B_{4}$.

The study of pattern avoidance on the hyperoctahedral group has been carried out by many authors in terms of signed patterns. If $\pi \in B_{n}$ and $\tau \in B_{k}, k \leqslant n$, we say that $\pi$ contains the pattern $\tau$ if there exists a sequence of indices $1 \leqslant i_{1}<i_{2}<\cdots<i_{k} \leqslant n$ such that two conditions hold:

- $\left|\pi\left(i_{1}\right)\right| \ldots\left|\pi\left(i_{k}\right)\right|$ is order-isomorphic to $|\tau|$,

- $\pi\left(i_{j}\right)$ has the same sign as $\tau_{j}$ for $1 \leqslant j \leqslant k$.

We say that $\pi$ avoids $\tau$ if $\pi$ does not contain $\tau$. For example, the signed permutation $\pi=6 \overline{1} 5 \overline{3} \overline{2} 4$ avoids the pattern $\tau=\overline{2} 1$ while it contains the pattern $\tau^{\prime}=2 \overline{1}$.

Signed pattern avoidance has received a lot of attention in recent years (see [10], [14], and [17]). In [17, Corollary 5.6], Stembridge characterized the set $T_{n}$ of so-called fully commutative top elements in $B_{n}$ as the subset of elements in $B_{n}$ that avoid the six signed patterns

$$
321, \quad \overline{3} 21, \quad 32 \overline{1}, \quad \overline{3} 2 \overline{1}, \quad 1 \overline{2}, \quad \overline{1} \overline{2} .
$$

Stembridge does not consider the subset of involutory elements in $T_{n}$. In the next proposition, we show that the image of $\mathcal{S}_{2 n}^{C}(321)$ under $\Theta$ is precisely the set $T_{n}$. This result appears in [7] in a slightly different form. 
Proposition 11. $\Theta\left(\mathcal{S}_{2 n}^{C}(321)\right)=T_{n}$.

Proof. Consider a permutation $\pi \in \mathcal{S}_{2 n}^{C}(321)$. Straightforward considerations imply that $\Theta(\pi)$ must avoid the six signed patterns in (8), since every occurrence of one of them in $\Theta(\pi)$ yields an occurrence of 321 in $\pi$.

Conversely, suppose that the permutation $\pi$ contains an occurrence $c b a$ of 321 . We want to show that $\Theta(\pi)$ contains at least one of the six patterns above. Denote by $j, k$, and $l$ the positions in $\pi$ of $c, b$, and $a$, respectively. Note that $c>b>a$ and $j<k<l$. Without loss of generality (due to the fact that $\pi$ is centrosymmetric), we can assume that either

i) $j, k, l>n$, or

ii) $j \leqslant n<k, l$.

In both cases, if $b, a \leqslant n$, then $\Theta(\pi)$ contains $\overline{1} \overline{2}$, so we will assume that $b>n$. Consider first case i). If $c, b, a>n$, then $\Theta(\pi)$ contains 321. Suppose that $c, b>n \geqslant a$. If $b<2 n+1-a$, then $\Theta(\pi)$ contains $1 \overline{2}$, while if $b>2 n+1-a$, then $\Theta(\pi)$ contains $32 \overline{1}$ (note that $b \neq 2 n+1-a$ since the centrosymmetric condition forces $2 n+1-a$ to appear in a position less than $n$ ).

Consider now case ii). Set $d=2 n+1-c$, and note that position of $d$ in $\pi$ is greater than $n$. If $c, b, a>n$, then $\Theta(\pi)$ contains either $\overline{3} 21$ (if $d$ precedes $b$ in $\pi$ ) or $1 \overline{2}$ (otherwise). If $c, b>n \geqslant a$, then $\Theta(\pi)$ contains either $\overline{3} 2 \overline{1}$ (if $d$ precedes $b$ in $\pi$ ) or $1 \overline{2}$ (otherwise). This completes the proof.

Many definitions of the descent set and of the major index of a signed permutation can be found in the literature (see e.g. [4], [5, and [11]). The notions of $\mathrm{Des}^{+}$and $\mathrm{maj}^{+}$are related with the analogous statistics $\operatorname{Des}_{B}$ and maj introduced in [5] as follows: if $\pi \in \mathcal{S}_{2 n}^{C}$, then $\operatorname{Des}_{B}(\Theta(\pi))=\left\{n-x \mid x \in \operatorname{Des}^{+}(\pi)\right\}$ and maj $(\Theta(\pi))=n \cdot \operatorname{des}^{+}(\pi)-\operatorname{maj}^{+}(\pi)$.

\section{Descents and fixed points in 321-avoiding involutions}

The distribution of the major index over the set of 321-avoiding involutions was studied in [1, where it is shown to be given by the $q$-analogue of the central binomial coefficients. This is proved by constructing a bijection, as stated in the following theorem, which is the main result from [1].

Theorem 12 ([1, Theorem 3.4]). There is a bijection between $\mathcal{I}_{n}(321)$ and $\mathcal{Y}_{\left\lfloor\frac{n}{2}\right\rfloor,\left\lceil\frac{n}{2}\right\rceil}$ that maps Des to HD.

Let $\operatorname{fp}(\pi)$ be the number of fixed points of $\pi$, that is, elements $i$ such that $\pi(i)=i$. Using the bijection from Lemma 10 , we can generalize Theorem 12 as follows.

Theorem 13. Let $b \geqslant a \geqslant 0$. There is a bijection

$$
\theta:\left\{\pi \in \mathcal{I}_{a+b}(321): \mathrm{fp}(\pi) \geqslant b-a\right\} \longrightarrow \mathcal{Y}_{a, b}
$$

such that if $\theta(\pi)=\lambda$, then $\operatorname{Des}(\pi)=\operatorname{HD}(\lambda)$. 
For $b=a$ and $b=a+1$ we recover Theorem 12 .

Proof. Following the same idea from the proof of Theorem 12 given in [1], the first step of the construction is the Robinson-Schensted correspondence, which gives a bijection between $\mathcal{I}_{a+b}(321)$ and standard Young tableaux with $a+b$ boxes and at most two rows. We claim that the number of fixed points of $\pi \in \mathcal{I}_{a+b}(321)$ equals the difference in size of the two rows of the corresponding standard Young tableau. To see this, first note that since $\pi$ is an involution, its excedances and antiexcedances (i.e., positions $i$ such that $\pi(i)<i$ ) are naturally paired up by symmetry. It follows that the excedance values and fixed points of $\pi$ form a longest increasing subsequence (such a sequence is increasing because $\pi$ avoids 321, and it is longest because at most one element of each pair excedance-antiexcedance can be in it). By Schensted's theorem [12, the length of a longest increasing sequence is the size of the first row of the tableau. Thus, the size of the second row has to be equal to the number of antiexcedances, which equals the number of excedances. It follows that the difference between the sizes of the rows of the tableau is $\mathrm{fp}(\pi)$, as claimed. Alternatively, this fact be easily derived from [8].

From the standard Young tableau, we construct a path in $\mathcal{A}_{a+b}$ whose $i$-th step is an $N$ if $i$ is in the top row of the tableau, and an $E$ otherwise. By construction, this path does not go below the diagonal $y=x$, and its number of $N$ steps minus its number of $E$ steps equals $\mathrm{fp}(\pi)$, and so it has $(a+b+\mathrm{fp}(\pi)) / 2 N$ steps and $(a+b-\mathrm{fp}(\pi)) / 2 E$ steps. Additionally, $\operatorname{Des}(\pi)$ becomes the peak set of this path, since $i$ is a descent of $\pi$ if and only if $i$ is in the top row of the corresponding tableau and $i+1$ is in the bottom row.

The next step in the construction consists in matching $N$ s and Es that face each other in the path, in the sense that the line segment from the midpoint of $N$ to the midpoint of $E$ has slope 1 and stays below the path. Thinking of the $N$ s as opening parentheses and the Es as closing parentheses, the matched parentheses properly close each other. Note that every $E$ step gets matched with an $N$ step, but there $\operatorname{are} \operatorname{fp}(\pi)$ unmatched $N$ steps. Construct a new path $P$ by changing the leftmost $(\operatorname{fp}(\pi)+b-a) / 2$ unmatched $N$ steps into $E$ steps. The resulting path has $a N$ steps and $b E$ steps, so $P \in \mathcal{Y}_{a, b}$. This step is a bijection between paths in $\mathcal{A}_{a+b}$ not going below $y=x$ and having $\operatorname{fp}(\pi) \geqslant b-a$ unmatched steps, and $\mathcal{Y}_{a, b}$. It has the property that it preserves the positions of the peaks, and so $\operatorname{Des}(\pi)=\operatorname{Peak}(P)$. The inverse of this step is obtained by matching $N \mathrm{~s}$ and $E$ s that face each other in the path in $\mathcal{Y}_{a, b}$, and then changing all the unmatched $E \mathrm{~s}$ (which necessarily come before the unmatched $N \mathrm{~s}$, and of which there is at least $b-a$ of them) into $N \mathrm{~s}$.

Finally, the last step is the bijection $g$ from Lemma 10 applied to $P$. The composition of these bijections produces a path $\lambda:=g(P) \in \mathcal{Y}_{a, b}$ with $\operatorname{Des}(\pi)=\operatorname{HD}(\lambda)$.

Theorem 12 is used in [1] to prove that $\sum_{\pi \in \mathcal{I}_{n}(321)} q^{\operatorname{maj}(\pi)}=\left(\begin{array}{c}n \\ \left\lfloor\frac{n}{2}\right\rfloor\end{array}\right)_{q}$. We now get a refinement of this result with respect to the number of fixed points.

Corollary 14. For $b \geqslant a \geqslant 0$,

$$
\sum_{\substack{\pi \in \mathcal{I}_{a+b}(321) \\
\mathrm{fp}(\pi) \geqslant b-a}} q^{\operatorname{maj}(\pi)}=\left(\begin{array}{c}
a+b \\
a
\end{array}\right)_{q} .
$$


Consequently, if $\ell \equiv n \bmod 2$, then

$$
\sum_{\substack{\pi \in \mathcal{I}_{n}(321) \\
\operatorname{fp}(\pi)=\ell}} q^{\operatorname{maj}(\pi)}=\left(\begin{array}{c}
n \\
\frac{n-\ell}{2}
\end{array}\right)_{q}-\left(\begin{array}{c}
n \\
\frac{n-\ell}{2}-1
\end{array}\right)_{q}
$$

Proof. Applying the bijection from Theorem 13 and using that $\operatorname{maj}(\pi)=\sum_{i \in \operatorname{Des}(\pi)} i$ and $|\lambda|=\sum_{i \in \mathrm{HD}(\lambda)} i$, we have

$$
\sum_{\substack{\pi \in \mathcal{I}_{a+b}(321) \\
\mathrm{fp}(\pi) \geqslant b-a}} q^{\operatorname{maj}(\pi)}=\sum_{\lambda \in \mathcal{Y}_{a, b}} q^{|\lambda|}=\left(\begin{array}{c}
a+b \\
a
\end{array}\right)_{q} .
$$

For the second formula, note that by the symmetry between excedances and anitiexcedances, the number of fixed points of $\pi \in \mathcal{I}_{n}(321)$ has the same parity as $n$. Now take $a+b=n$ and subtract the case $b-a=\ell+2$ from the case $b-a=\ell$ in the first formula.

It is shown in [1] that

$$
\sum_{\substack{\pi \in \mathcal{I}_{n}(321) \\
\operatorname{des}(\pi)=k}} q^{\operatorname{maj}(\pi)}=q^{k^{2}}\left(\begin{array}{c}
\left\lceil\frac{n}{2}\right\rceil \\
k
\end{array}\right)_{q}\left(\begin{array}{c}
\left\lfloor\frac{n}{2}\right\rfloor \\
k
\end{array}\right)_{q} .
$$

The refinement that keeps track of the number of fixed points is the following.

Corollary 15. For $b \geqslant a \geqslant 0$,

$$
\sum_{\substack{\pi \in \mathcal{I}_{a+b}(321) \\
\mathrm{fp}(\pi) \geqslant b-a \\
\operatorname{des}(\pi)=k}} q^{\operatorname{maj}(\pi)}=q^{k^{2}}\left(\begin{array}{l}
a \\
k
\end{array}\right)_{q}\left(\begin{array}{l}
b \\
k
\end{array}\right)_{q}
$$

Consequently, if $\ell \equiv n \bmod 2$, then

$$
\sum_{\substack{\pi \in \mathcal{I}_{n}(321) \\
\mathrm{fp}(\pi)=\ell \\
\operatorname{des}(\pi)=k}} q^{\operatorname{maj}(\pi)}=q^{k^{2}}\left[\left(\begin{array}{c}
\frac{n-\ell}{2} \\
k
\end{array}\right)_{q}\left(\begin{array}{c}
\frac{n+\ell}{2} \\
k
\end{array}\right)_{q}-\left(\begin{array}{c}
\frac{n-\ell}{2}-1 \\
k
\end{array}\right)_{q}\left(\begin{array}{c}
\frac{n+\ell}{2}+1 \\
k
\end{array}\right)_{q}\right] .
$$

Proof. By the bijection in Theorem 13, the left hand side of the first formula is the generating polynomial with respect to area for Young diagrams in $\mathcal{Y}_{a, b}$ whose hook decomposition has $k$ hooks. The right hand side is obtained by decomposing such diagrams in three pieces: the top-left $k \times k$ square, which contributes $q^{k^{2}}$; a partition in $\mathcal{Y}_{k, a-k}$, which contributes $\left(\begin{array}{l}a \\ k\end{array}\right)_{q}$, and a partition in $\mathcal{Y}_{b-k, k}$, which contributes $\left(\begin{array}{l}b \\ k\end{array}\right)_{q}$.

The second formula follows immediately substituting $a+b=n$ and subtracting the case $b-a=\ell+2$ from the case $b-a=\ell$. 


\section{References}

[1] M. Barnabei, F. Bonetti, S. Elizalde, M. Silimbani, Descent sets on 321-avoiding involutions and hook decompositions of partitions, J. Combin. Theory Ser. A 128 (2014), 132-148.

[2] M. Barnabei, F. Bonetti, M. Silimbani, The Eulerian distribution on centrosymmetric involutions, Discrete Math. Theor. Comput. Sci. 11 (2009), 95-115.

[3] M. Barnabei, F. Bonetti, M. Silimbani, The Eulerian numbers on restricted centrosymmetric permutations, Pure Math. Appl. 21 (2010), 99-118.

[4] R. Biagioli, F. Jouhet, P. Nadeau, Combinatorics of fully commutative involutions in classical Coxeter groups, Discrete Math. 338 (2015), 2242-2259.

[5] R. Biagioli, J. Zeng, On some analogues of descent numbers and major index for the hyperoctahedral group, Sém. Lothar. Combin. 61A (2009/11), Art. B61Ak, 13 pp.

[6] A.B. Cruse, Some combinatorial properties of centrosymmetric matrices, Linear Algebra Appl. 16 (1977), 65-77.

[7] E.S. Egge, Restricted symmetric permutations, Ann. Comb. 11 (2007) 405-434.

[8] S. Elizalde, I. Pak, Bijections for refined restricted permutations, J. Combin. Theory Ser. A 105 (2004), 207-219.

[9] D.E. Knuth, The art of computer programming, Vol. 3: Sorting and searching, Addison-Wesley, Reading, MA (1998).

[10] T. Mansour, J. West, Avoiding 2-letter signed patterns, Sém. Lothar. Combin 49 (2002/04), Art. B49a, 11 pp. (electronic).

[11] V. Reiner, Signed permutation statistics, European J. Combin. 14 (1993), 553-567.

[12] C. Schensted, Longest increasing and decreasing subsequences, Canad. J. Math 13 (1961), 179-191.

[13] M.P. Schützenberger, Quelques Remarques sur une Construction de Schensted, Math. Scand. 12 (1963), 117-128.

[14] R. Simion, Combinatorial statistics on type-B analogue of non crossing partitions and restricted permutations, Electron. J. Combin 7 (2000), \#P9.

[15] R. Simion and F.W. Schmidt, Restricted Permutations, European J. Combin. 6 (1985), 383-406.

[16] R. Stanley, Algebraic Combinatorics: Walks, Trees, Tableaux, and More, Springer, 2013.

[17] J.R. Stembridge, Some combinatorial aspects of reduced words in finite Coxeter groups, Trans. Amer. Math. Soc. 349 (1997), 1285-1332. 